\title{
NISTATINA COMO INHIBIDOR DE CAVEOLAS: TRATAMIENTO PREVENTIVO PARA EL CONTAGIO POR COVID-19
}

\author{
José Palacio Angulo ${ }^{1} *$ \\ ${ }^{1,2,3}$ Corporacion Universitaria Minuto de Dios, UNIMINUTO. Barranquilla, Colombia \\ *Autor de correspondencia: jose.palacio@uniminuto.edu.co
}

Recibido Abril 2020; Aceptado Junio 2020

\begin{abstract}
Resumen - En el mundo, los antivirales y vacunas efectivo contra el COVID 19, aún se encuentran en fase de estudio y su alta demanda y alto costo, puede ser barrera para que llegue a todos los pacientes que la necesiten, en especial a los países en desarrollo; este trabajo tiene como objetivo formular una hipótesis de administrar un medicamento que se encuentre disponible actualmente en los países desarrollado, que prevenga la enfermedad por contagio de COVID19 o sus complicaciones; a través del método praxeológico y concluye la posibilidad de usar estratégicamente la nistatina en forma de suspensión oral para inhibir la formación de caveolas en las membranas celulares y así impedir la entrada del nuevo coronavirus en las células de la mucosa oral, retrofaringea y esofágica e incluso en mucosa gastrointestinal, el cual no podría replicarse en estas mucosas de forma eficiente, y su carga viral no sería suficiente para replicarse en otros tejidos, previniendo la enfermedad por contagio de COVID19 o disminuyendo su posibilidad de SARS, o fallas multiorgánicas.
\end{abstract}

Palabras Clave: Infecciones por Coronavirus, Caveolina 1, Pandemias, Nistatina, Virus del SARS.

\section{NYSTATIN AS A CAVEOLE INHIBITOR: PREVENTIVE TREATMENT FOR TRANSMISSION BY COVID-19}

\begin{abstract}
In the world, effective antivirals and vaccines against COVID 19 are still in the study phase and their high demand and high cost can be a barrier to reaching all patients who need them, especially in developing countries; This work aims to formulate a hypothesis to administer a drug that is currently available in developed countries, which prevents the disease due to COVID19 infection or its complications; through the praxeological method and concludes the possibility of strategically using nystatin in the form of oral suspension to inhibit the formation of caveole in cell membranes and thus prevent the entry of the new coronavirus into the cells of the oral, retropharyngeal and esophageal mucosa and even in gastrointestinal mucosa, which could not replicate in these mucosae efficiently, and its viral load would not be sufficient to replicate in other tissues, preventing the disease due to COVID19 contagion or reducing its possibility of SARS, or multi-organ failure.
\end{abstract}

Palabras Clave: Coronavirus Infections, Caveolin 1, Pandemics, Nystatin, SARS Virus. 


\section{Introducción}

Nistatina como inhibidor de caveolas: tratamiento preventivo para el contagio por COVID-19

A finales de diciembre de 2019, ocurrió un brote inexplicable de neumonía masiva en Wuhan, China, que atrajo la atención de las autoridades de salud. El 31 de diciembre, el Centro Chino para el Control y la Prevención de Enfermedades envió un equipo de respuesta rápida a Wuhan. (Novel Coronavirus Pneumonia Emergency Response Epidemiology, 2020), y el 7 de enero de 2020, identificaron un nuevo tipo de virus de la familia Coronaviridae, denominado nuevo coronavirus, COVID-19, como agente causante del brote, en el cual sus enfermos tenían en común una exposición a un mercado de marisco, pescado y animales vivos en la ciudad de Wuhan (Calvo, López-Hortelano, de Carlos Vicente, Vásquez y Grupo de trabajo de la Asociación Española de Pediatría, 2020).

En vista de la alta velocidad y la escala de la transmisión del nuevo coronavirus, COVID-19, la Organización Mundial de la Salud, en el mes de marzo del 2020, declaró que el brote del nuevo coronavirus COVID-19, como una pandemia; e insto a los países del mundo a promover la salud pública con un enfoque basado en la participación de todo el gobierno y de toda la sociedad, (OMS, 2020).

Pronto, se observó que el nuevo coronavirus podría causar enfermedad SARS, síndrome respiratorio agudo grave, como las cepas específicas de coronavirus como el SARS-CoV1, aunque su mecanismo no es bien conocido, Weiss y Navas-Martin, S. (2005), proponen un modelo de enfermedad de SARS, que consta de tres fases: replicación viral, hiperactividad inmune y destrucción pulmonar. La patología pulmonar por SARS se ha asociado con daño alveolar difuso, proliferación de células epiteliales y un aumento de macrófagos.

Los infiltrados multinucleados de células gigantes de origen macrófago u epitelial se han asociado con una supuesta formación similar al sincitio que es característica de muchas infecciones por coronavirus. La linfopenia, la hemofagocitosis en el pulmón y la atrofia de la pulpa blanca del bazo observada en pacientes con SARS recuerdan a los reportados para la enfermedad mortal del subtipo H5N1 del virus de la influenza en 1997. Sorprendentemente, la presencia de hemofagocitosis apoya una desregulación de citoquinas. Se considera ampliamente que el SARS es una neumonía viral. Sin embargo, los pacientes con SARS también pueden presentar síntomas gastrointestinales y atrofia esplénica y linfadenopatía. La diarrea es un hallazgo muy frecuente en pacientes con SARS (30 a 40\% de los pacientes). El SARS-CoV se replica en los enterocitos, con una interrupción mínima de la arquitectura intestinal. Se ha especulado que la ausencia de inflamación intestinal es el resultado de la regulación positiva del factor de crecimiento transformante $\beta$ y una respuesta celular del huésped antiapoptótico en las células epiteliales intestinales.

Hallazgos basados en autopsias de pacientes con SARS propusieron que el SARS es una enfermedad sistémica con diseminación extrapulmonar generalizada, que resulta en la eliminación de virus en secreciones respiratorias, heces, orina e incluso sudor (Farcas, Poutanen, Mazzulli, Willey, Butany, Asa, Faure, Akhavan, Low y Kain, 2005). Igualmente, se ha observado que principal complicación en el paciente contagiado por el nuevo coronavirus COVID-19, es el síndrome de distress respiratorio agudo, y ocurre frecuentemente, seguidamente después de la sensación de disnea entre el día 6 a 8 de los primeros síntomas. Otras complicaciones incluyen la arritmia, cardiomiopatía, fallo renal agudo, y shock (Villafranca, Albelo, Moreno, Pérez y Herrera, 2020).

Hasta el momento, según Shervani, Khan y Qazi (2020), los medicamentos que han demostrado accion directa en contra del nuevo coronavirus, COVID-19, son el Remdesivir y Avifavir o Favipiravir, los cuales 
Nistatina como inhibidor de caveolas: tratamiento preventivo para el contagio por COVID-19 son inhibidores de la polimerasa de ARN viral; asimismo las compañías farmacéuticas de todo el mundo, entre ella Moderna Inc., AstraZeneca PLC, Johnson \& Johnson y Sanofi y Merck \& Co, están trabajando horas extras para desarrollar la vacunas contra el COVID-19, lo antes posible, pero tanto los antivirales como vacunas mencionadas, aún se encuentran en fase de estudio y su alta demanda y alto costo, puede ser barrera para que llegue a todos los pacientes que la necesiten, en especial a los países en desarrollo.

Mientras, llega de forma universal y accesible la vacuna o un tratamiento eficaz contra el COVID-19 a los países en desarrollo, es importante investigar entre los medicamentos que se dispone en ellos, para para prevenir la enfermedad por contagio de COVID-19 o sus complicaciones. Por lo cual este trabajo tiene como objetivo de formular la hipótesis de administrar un medicamento que se encuentre disponible actualmente en los países desarrollados, para prevenir la enfermedad por contagio de COVID-19 o sus complicaciones

\section{Materiales y Métodos}

Se utiliza para la presente investigación, el método praxeológico de la Corporacion Universitaria Minuto de Dios. (2014), que se basa en 4 momentos (Palacio-Angulo et al., 2018): el primero el VER, que se basa en la observación el quehacer y sus procesos, donde se revisara la documentación respecto al nuevo coronavirus COVID-19 y sobre los inhibidores de caveolas en el proceso de endocitosis; después se aplicara el momento de JUZGAR, entendido como el análisis comprensivo de lo que se ha observado, en su comparación con diferentes teorías y modelos; lo que conlleva al ACTUAR, realizando una intervención con un plan estratégico de acción y de mejora de la práctica plasmando el análisis de los visto y juzgado y llegando al momento final la DEVOLUCION CREATIVA que evalúa la intervención e imagina otros mundos posibles, modelizando el proceso realizado, de modo que pueda ser socializado y/o replicado por otros, a través de las conclusiones dadas.

\section{Resultados}

a) Análisis del mecanismo de infestación del COVID-19

El nuevo coronavirus COVID-19, pertenece a la familia de los coronaviridae, de la subfamilia Orthocoronavirinae, género Coronavirus y al subgénero Beta-coronavirus, (Reina, 2020). Además, el nuevo coronavirus COVID-19 un virus ARN positivo de cadena única, de forma esférica con proteínas de superficie en forma de espigas (proteínas S) que le dan el aspecto de corona (Lobato, López, Alba, Anaya, García y Barrios, 2020).

Una vez que el COVID-19, se encuentra en contacto con las células de la mucosa nasal, oral o faríngea, se plantea al igual que para el SARSCoV-1, que la proteína $S$ de la superficie del virus es la que se une a este receptor ACE2 o Enzima convertidora de Angiotensina2, ECA2 de la celula huésped, pero con mayor afinidad; (Tian, Li, Huang, Xia, Lu, Shi, Lu, Jiang, Yang, Wu y Ying, 2020); donde el RBD de la subunidad S1 de la proteína S actúa como mediadora para unir el virus con los receptores celulares, mientras que la subunidad S2 es la mediadora de los eventos de fusión entre la membrana viral y celular (Accinelli, Xu, Yachachin-Chávez, Cáceres-Pizarro, Tafur-Bances, Flores-Tejada y Paiva-Andrade, (2020). 
Nistatina como inhibidor de caveolas: tratamiento preventivo para el contagio por COVID-19 La evidencia actual sugiere que el modo de entrada puede variar entre los virus y los tipos de células huésped, y puede incluir endocitosis de caveolas dependiente de caveolina, y/o clatrina, mecanismo independiente que involucra balsas lipídicas, asimismo se propone la fusión en la superficie celular, mediada por la proteína M viral como un posible mecanismo de entrada, esto sugiere que el COVID-19, puede ser endocitosado por más de una vía, dependiendo de la línea celular que contagie (Glebov, 2020).

Una vez el virus se encuentra en el interior de la célula, queda expuesta la proteína N que se encontraba en el interior del nuevo coronavirus COVID-19, la cual envolvía el ARN viral, el cual se libera en el citoplasma celular. (Fernández-Rodríguez, Casas, Culebras, Morilla, Cohen y Alberola, 2020).

El ARN viral es de cadena simple positiva que mide de 26,2 a 31,7 kb., genoma viral que está compuesto por seis a diez marcos de lectura abiertos (ORF, por sus siglas en inglés), la cual codifica su información genética (Aguilar Ramírez, P., Enriquez Valencia, Quiroz Carrillo, Valencia Ayala, de León Delgado y Pareja Cruz, 2020).

Después de liberado en el citoplasma el ARN viral, el nuevo coronavirus COVID-19 emplea una maquinaria de múltiples subunidades para la replicación y la transcripción de su material genético, un conjunto de proteínas no estructurales (nsps) producidas como productos de escisión de las poliproteínas virales ORF1a y ORF1ab se ensambla para facilitar la replicación y transcripción viral. Un componente clave, es la ARN polimerasa dependiente de ARN (RdRp), también conocida como nsp12, esta estructura, como en otras ARN polimerasas, las rutas de entrada de plantilla de cebador, es entrada de NTP y salida de hebra naciente están cargadas positivamente y son accesibles al solvente, y convergen en una cavidad central donde los motivos de RdRp median la síntesis de ARN viral dirigida por plantilla y por lo tanto juega un papel central en el ciclo de replicación y transcripción del virus COVID-19, posiblemente con la ayuda de nsp7 y nsp8 como cofactores. (Gao, Yan, Huang, Liu, Zhao, Cao, Wang, Sun, Ming, Zhang, Ge, Zheng, Zhang, Wang, Zhu, Zhu, Hu, Hua, Zhang, ... Rao, 2020).

Asimismo, las proteínas estructurales (S), (M) y (E); y las proteínas accesorias, expresadas a partir de otros segmento del ARN viral, son elaboradas en las membranas del retículo endoplasmático (RE) y posteriormente trasportadas al complejo de Golgi donde serán ensambladas junto con la nucleocápside, conformada por la Proteína N y ARN viral, para producir nuevas partículas víricas, las que serán exportadas hacia la membrana plasmática celular en forma de vesículas (Pastrian-Soto, 2020).

Al mismo tiempo, la proteína E, unidas a otras proteínas accesorias, conducen a la aparición de canales llamados virioporos. Los cuales son orificios que favorecen al desequilibrio de iones entre ambos lados de la membrana del retículo endoplasmático, que origina un proceso de apoptosis, autofagia y muerte celular, liberándose miles de virus que se encuentran en la membrana plasmática celular en forma de vesículas, hacia el espacio extracelular, y cada uno con capacidad de infectar a una nueva célula (Torres, Cutiño, Prieto y González, 2020).

b) Inhibidores de la caveolas

En la biogénesis de las caveolas. el colesterol tiene un papel muy importante, por lo tanto, si se aplica a las células, quelantes de colesterol como nistatina, filipina o ciclodextrina, no se produce la formación de caveolas, (Maza, 2008) 
Nistatina como inhibidor de caveolas: tratamiento preventivo para el contagio por COVID-19 También entre los inhibidores de las caveolas, se encuentra Forbol 12-miristato 13-acetato (PMA), que inhibe la endocitosis dependiente de caveolas al fosforilar caveolina (Báez, 2012), a diferencia de la nistatina que es un agente secuestrante de colesterol, que interrumpe la formación e internalización de la balsa lipídica e inhibe la endocitosis dependiente de caveolas (Stuart, Eustace, McKee y Brown, 2002)

Y comenta Mayoral Moñibas (2007), que aunque las caveolas y las balsas lipídicas son microdominios enriquecidos en colesterol, estudios varios han demostrado que la manipulación del colesterol celular también afecta a la formación de vesículas endocíticas recubiertas de clatrina y de vesículas sinápticas, que son estructuras diferentes a las balsas lipídicas o las caveolas

\section{c) Análisis de la Nistatina}

La Nistatina es un medicamento importante de un grupo variado de antimicrobianos antifúngicos altamente insaturados producido en los cultivos de Streptomyces noursei. Según su estructura química se la incluye dentro del grupo denominado antimicrobianos antifúngicos de tipo poliénico y puede presentar tres formas polimórficas, denominadas Tipo A, Tipo B y Tipo C; siendo la mas común la tipo A y B y pueden interconvertirse por efecto de condiciones ambientales. (Lescano, Pettigrosso y Llabot, 2014).

Es un medicamento que se utiliza de forma local sobre piel y mucosa, y debido a que no se absorbe por parte de ella, no tiene efectos sistémicos al utilizarse por esta vía, por lo que sus diversas presentaciones farmacéuticas son pomadas, cremas y talcos para su aplicación tópica en piel, cremas y óvulos para tratar la infecciones vaginales y suspensión para infecciones orales y faríngeas (Pascal y Maritsa, 2015).

Desde que se descubrió la nistatina, en el Health Laboratory del estado New York, se ha probado en su presentación de suspensión oral de 100.000UI en un ml, y administrándose en forma segura via oral tanto a neonato, lactante, niños, adultos e imunocomprometidos, indicándole que al tomar el fármaco lo recorran por su cavidad oral y después deglutirlo para una mayor acción antifungica en la mucosa oral, retrofaringea y esofágica e incluso en mucosa gastrointestinal; y salvo a un poco de sabor amargo y a veces nauseas, son infrecuentes los efectos adversos reportados. (Brunton, Lazo y Parker, 2007).

Lo anterior, sucede dado que la absorción sistémica de nistatina es muy escasa y la poca cantidad que pueda absorberse se desactiva en un 95\% por el metabolismo, siendo su eliminación renal, además no presenta metabolismo importante a nivel hepático, por lo que no presenta interacciones importantes con fármacos ni alimentos (Benavent Núñez, 2019).

Asimismo, Gómez (2015), afirma que la nistatina en suspensión oral es seguro administrarlo en pacientes embarazadas como en periodo de lactancia; pero, García y Medrano (2010), nos recuerda a que la Food And Drugs Administration (FDA) en su clasificación de productos farmacológicos en el embarazo, clasifica la nistatina en C: Riesgo fetal desconocido; no hay estudios adecuados en seres humanos.

Según, Brunton, Lazo y Parker (2007), la nistatina oral se presenta suspensión de 100.000 U.I. en 1ml. y su posología es en recién nacidos y lactantes con bajo peso al nacer: es de $1 \mathrm{ml}$. cada 6 horas, en lactantes: 2ml. cada 6 horas y niños mayores de 1 año y adultos: $4 \mathrm{ml}$. a $6 \mathrm{ml}$ cada 6 horas.

El mecanismo de acción farmacológica de la nistatina, al igual que la anfotericina $\mathrm{B}$, es una molécula antifilica y antifoterico, que se incrusta en las membrana bicapas lipídica celular de los hongos, uniéndose 
Nistatina como inhibidor de caveolas: tratamiento preventivo para el contagio por COVID-19 al ergosterol, molécula similar al colesterol, permitiendo la salida de potasio de su interior y causando la muerte celular. (Fernández Campos, 2012).

Hay que señalar, además, que la nistatina forma un complejo con él colesterol dentro de la membrana celular que interfieren en la composición y estructura de lipid-rafts o caveolas, convirtiéndose en un compuesto que bloquea esta vía de entrada de los virus a las células, y se encuentran estudios que demuestran que la nistatina presentan una importante actividad virucida contra los 4 serotipos del virus de Dengue (Carro, 2015).

También, comentan Gutiérrez y López (2010) que existen varios virus que utilizan la endocitosis mediadas por caveolas entrando por la ruta dependiente de caveolina, tales como el virus de la leucemia murina, ecovirus y SV40; y que la infectividad de ellos se ve disminuida cuando se trata a las células con drogas como la nistatina, que inhiben esta vía.

Asimismo, Báez (2012) argumenta que la nistatina, es un antimicrobiano que en su mecanismo de acción, se une a esteroides, inhibiendo la endocitosis dependiente de caveolas al eliminar el colesterol de la membrana, que resulta imprescindible para el mantenimiento de las caveolas y su separación de las membranas, y demostró en experimento in vitro en células HeLa y COS-7 que es un medicamento inhibidor de la endocitosis sobre la fusión inducida por las glicoproteínas de los virus respiratorios, donde los resultados mostraron entre 49\% y 93\% de efecto inhibidor de endocitosis sobre la formación de sincitios inducida por el virus de la enfermedad de Newcastle (NDV) en células COS-7 y HeLa. Además se observó en la preincubación de las células diana con nistatina y provocó una reducción de la entrada del NDV en la célula hospedadora, permaneciendo el virus principalmente localizado en el exterior de la membrana celular de las células tratadas y no en el interior y este efecto estuvieron presentes durante todo el ensayo; también se constató que la nistatina bloquea parcialmente la infectividad y la fusión del NDV lo que confirmaría que existen simultáneamente dos rutas de entrada para este virus, pero hay bloqueo completo de la infectividad y la fusión del Virus Respiratorio Sincitial (RSV), ya que en virus que sólo usen endocitosis el bloqueo sería total.

Asimismo en experimento in vitro ejecutado por Anderson, Chen y Norkin (1996), se demostró que la nistatina aunque no perjudicó la unión de Virus del Simio 40 (SV40) en células humanas, si bloqueó la división del virus en los complejos enriquecidos con la proteína estructural de caveolina, debido que la nistatina se unió al colesterol, lo que altero selectivamente las caveolas de la membrana celular.

También en estudio realizado in vitro por Pho, Ashok y Atwood (2000), se observó que la nistatina inhibió la entrada infecciosa a través de endocitosis mediada por caveolas del Virus del Simio 40 (SV40), a las células gliales humanas, pero no encontró que la nistatina tuviera algún efecto sobre la endocitosis dependiente de clatrina.

\section{Conclusiones}

A base de los resultados, se puede una dar una devolución creativa de la formulación de la hipótesis de que se puede usar estratégicamente la nistatina en forma de suspensión oral para inhibir la formación de caveolas en las membranas celulares y así impedir la entrada del nuevo coronavirus en las células de la mucosa oral, retrofaringea y esofágica e incluso en mucosa gastrointestinal, para prevenir la enfermedad por contagio de COVID-19 o sus complicaciones. 
Nistatina como inhibidor de caveolas: tratamiento preventivo para el contagio por COVID-19 La dosis de administración de la nistatina en suspensión oral en la presentación 100.000 UI en 1 ml, para para prevenir la enfermedad por contagio de COVID-19 o las complicaciones de esta, sería la misma que se utiliza típicamente para el tratamiento de otras infecciones:

Recién nacidos y lactantes con bajo peso al nacer: 1 ml. cada 6 horas

Lactantes: 2 ml. cada 6 horas.

Niños mayores de 1 año y adultos: de 4 a 6 ml. cada 6 horas.

Una vez dada en la dosis señalada la nistatina impregna rápidamente la mucosa oral, faríngea, esofágica y gastrointestinal actuando inmediatamente sobre el área expuesta en primera instancia por el coronavirus COVID-19, el cual no podría replicarse en estas mucosas de forma eficiente, y su carga viral no sería suficiente para replicarse eficientemente en las células del sistema respiratorio, disminuyendo la posibilidad de SARS, o fallas multiorgánica por nuevo coronavirus COVID-19.

Entre las ventajas de usar la nistatina se encuentra:

- Su administración oral impregna rápidamente la mucosa oral, faríngea, esofágica y gastrointestinal actuando inmediatamente sobre el área expuesta en primera instancia por el nuevo coronavirus COVID-19.

- Con la administración en forma oral de la nistatina, su absorción en forma sistémica es casi nula por lo cual no hay efectos adversos secundarios significativos.

- Se puede administrar oralmente en forma segura en todas las edades, tanto a neonatos, lactantes, niños, adultos e imunocomprometidos.

- Se encuentra actualmente en los países en desarrollo, y en la mayoría es producido por sus laboratorios nacionales, lo que garantizan su suministro en altas cantidades

- Su valor económico es accesible y oscila entre 1 a 2 dólares la suspensión oral por 60 ml, aunque lo gobiernos de los países en desarrollo puede adquirirlo a un precio menor por unidad si realiza compras al por mayor del mismo, para su distribución en la comunidad.

Entre sus desventajas se encuentra:

- Debido a que se administrara en forma oral la nistatina, su absorción en forma sistémica es casi nula, y solo tendría efecto sobre el nuevo coronavirus COVID-19 en la área local y no sistémica.

- Para lograr el efecto planteado de la nistatina tendría que administrarse en los primeros días de exposición al nuevo coronavirus o antes de que esta ocurra.

- Debido a la clasificación C de la nistatina en la clasificación de productos farmacológicos en el embarazo de la FDA, debe restringirse su uso durante la gestación o administrarse después de evaluación riesgo/beneficio.

Para comprobación de la presente hipótesis se recomienda un estudio de cohortes, pero al llevar este tiempo, se sugiere también realizar un estudio de casos y controles, en el cual se compare un grupo de enfermos de COVID-19, que en su anamnesis tenga como antecedentes reciente la administración oral de nistatina reciente; con un grupo de enfermos de COVID-19 que en su anamnesis no tenga como antecedentes reciente la administración oral de nistatina reciente, y cada uno de los grupos con 2 subgrupos de casos severos y casos no severos. 
Nistatina como inhibidor de caveolas: tratamiento preventivo para el contagio por COVID-19 Asimismo, se recomienda seguir profundizando en el análisis de los procesos de infección de nuevo coronavirus COVID-19, para plantear nuevas formas de neutralización del mismo a través de medicamentos disponibles, mientras, llega de forma universal y accesible la vacuna o un tratamiento eficaz contra el COVID-19 a los países en desarrollo.

\section{Referencias}

Accinelli, R. A., Xu, C. M. Z., Yachachin-Chávez, J. M., Cáceres-Pizarro, J. A., Tafur-Bances, K. B., Flores-Tejada, R. G., y Paiva-Andrade, A. (2020). COVID-19: La pandemia por el nuevo virus SARS-CoV-2. Revista Peruana de Medicina Experimental y Salud Pública, 37(2).

Aguilar Ramírez, P., Enriquez Valencia, Y., Quiroz Carrillo, C., Valencia Ayala, E., de León Delgado, J., \& Pareja Cruz, A. (2020). Pruebas diagnósticas para la COVID-19: la importancia del antes y el después. Horizonte Médico (Lima), 20(2).

Anderson, H.A., Chen, Y., \& Norkin, L.C. (1996). Bound simian virus 40 translocates to caveolin-enriched membrane domains, and its entry is inhibited by drugs that selectively disrupt caveolae. Mol. Biol. Cell 7, 1825-1834.

Baez, J. H. (2012). Estudio de los receptores y mecanismos de entrada de los paramixovirus: virus respiratorio sincitial y virus de la enfermedad de Newcastle (Doctoral dissertation, Universidad de Salamanca).

Benavent Núñez, C. (2019). Desarrollo galénico de nuevas formulaciones de nistatina para uso en candidiasis oral (Doctoral dissertation, Universidad Complutense de Madrid).

Brunton, L., Lazo. J. y Parker. K. (2007). Goodman \& Gilman. Las bases farmacológicas de la terapéutica. 11ª ed. McGrawHill Interamericana Editores, México;

Calvo, C., López-Hortelano, M. G., de Carlos Vicente, J. C., Vasquez Martínez, J. L. y Grupo de trabajo de la Asociación Española de Pediatría (2020). Recomendaciones sobre el manejo clínico de la infección por el «nuevo coronavirus» SARSCoV2. In Anales de Pediatría. 2 (1) 1 -11.

Carro, A. C. (2015). La entrada de virus dengue a líneas celulares humanas en la infección primaria en ausencia o presencia de anticuerpos. Tesis Doctoral. Universidad de Buenos Aires.

Corporacion Universitaria Minuto de Dios. (2014). Proyecto Educativo Institucional (PEI) UNIMINUTO. Recuperado el 9 de Julio de 2017, de Portal Uniminuto: www.uniminuto.edu/documents/941377/1434225/Proyecto+Educativo+Institucional+2013.pdf/849a034b-2ee8-448c-9aa993e2cef4a317

Farcas, G. A., Poutanen, S. M., Mazzulli, T., Willey, B. M., Butany, J., Asa, S. L., Faure, P., Akhavan, P., Low, D. y Kain, K. C. (2005). Fatal severe acute respiratory syndrome is associated with multiorgan involvement by coronavirus. Journal of Infectious Diseases, 191(2), 193-197.

Fernández Campos, F. (2012). Nanoemulsiones de nistatina para el tratamiento de candidiasis muco-cutáneas. Tesis Doctoral. Universidad de Barcelona. http://diposit.ub.edu/dspace/bitstream/2445/34846/2/FFC_TESIS.pdf

Fernández-Rodríguez, A., Casas, I., Culebras, E., Morilla, E., Cohen, M. C., \& Alberola, J. (2020). COVID-19 y estudios microbiológicos post mortem. Revista Española de Medicina Legal. 46 (3) 127 - 138

Gao, Y., Yan, L., Huang, Y., Liu, F., Zhao, Y., Cao, L., Wang, T., Sun, Q., Ming, Z., Zhang, L., Ge, J., Zheng, L., Zhang, Y., Wang, H., Zhu, Y., Zhu, C., Hu, T., Hua, T., Zhang, B., ... Rao, Z. (2020). Structure of the RNA-dependent RNA polymerase from COVID-19 virus. Science, 368(6492), 779-782.

García, O. M. F., \& Medrano, M. G. C. (2010). Atención odontológica en la mujer embarazada. Archivos de investigación materno infantil, 2(2), 80-84.

IPSA SCIENTIA - Vol. 5 No $1-2020$ 
Nistatina como inhibidor de caveolas: tratamiento preventivo para el contagio por COVID-19

Glebov, O. O. (2020). Understanding SARS

Disponible en: https://doi.org/10.1111/febs.15369

Gómez, E. A. L. (2015). Embarazo y otros estados fisiológicos. En: Medicina en Odontología, 425 - 436

Gutiérrez, M., \& López, S. (2010). Mecanismos de entrada de virus: una manera de conocer a la célula. TIP Revista Especializada en Ciencias Químico-Biológicas, 13(1), 26-34.

Lescano, G. M., Pettigrosso, R. S., \& Llabot, J. M. (2014). Determinación de propiedades fisicoquímicas de Nistatina comercial empleando técnicas de caracterización de materiales. Revista mexicana de ciencias farmacéuticas, 45(2), 31-36.

Lobato, S. M., López, B. C. F., Alba, J. V. G., Anaya, C., García, J. P., \& Barrios, J. P. (2020). Pneumonia and SARS-CoV-2 Acute Respiratory Distress Syndrome in Mexico City. Medicina Crítica, 33(1), 68-72.

Mayoral Moñibas, R. M. (2007). Papel de la caveolina en proliferación y en regeneración hepática (Doctoral dissertation, Universidad Autónoma de Madrid).

Maza, P. K. (2008). Estudos sobre o envolvimento de "membrane rafts” e a ativação de quinases de células epiteliais durante a interação com paracoccidioides brasiliensis. Tesis de Maestria. Universidade Federal de Sao Paulo. http://repositorio.unifesp.br/bitstream/handle/11600/9604/PublicoTese\%2520Paloma\%2520Korehiza\%2520Maza\%2520versao\%2520final.pdf?sequence=1\&isAllowed=y

Novel Coronavirus Pneumonia Emergency Response Epidemiology. (2020). The epidemiological characteristics of an outbreak of 2019 novel coronavirus diseases (COVID-19) in China. Zhonghua liu xing bing xue za zhi= Zhonghua liuxingbingxue zazhi, 41(2), 145.

OMS (2020). Alocución de apertura del Director General de la OMS en la rueda de prensa sobre la COVID-19 celebrada el 11 de marzo de 2020. https://www.who.int/es/dg/speeches/detail/who-director-general-s-opening-remarks-at-the-mediabriefing-on-covid-19---11-march-2020

Palacio-Angulo, J., Ahumada-Villafañe, I., Orjuela, I. D., \& Posada-Lopez, J. (2018). Towards a strategic epidemiology of occupational safety and health. IPSA SCIENTIA: Revista Científica Multidisciplinaria, 3(1), 30-37.

Pascal, C., y Maritsa, L. (2015). Efecto antifúngico del aceite esencial del origanum vulgare (orégano) y cymbopogon citratus (hierba luisa), sobre cepas de cándida albicans en comparación con la nistatina estudio invitro (Bachelor's thesis, Quito: UCE).

Pastrian-Soto, G. (2020). Bases Genéticas y Moleculares del COVID-19 (SARS-CoV-2). Mecanismos de Patogénesis y de Respuesta Inmune. International journal of odontostomatology, 14(3), 331-337.

Pho, M. T., Ashok, A., y Atwood, W. J. (2000). JC virus enters human glial cells by clathrin-dependent receptor-mediated endocytosis. J.Virol. 74, 2288-2292.

Reina, J. (2020). El SARS-CoV-2, una nueva zoonosis pandémica que amenaza al mundo. Vacunas. 21 (1), 17-22.

Shervani, Z., Khan, I., y Qazi, U. Y. (2020). SARS-Cov-2 Delayed Tokyo 2020 Olympics: Very Recent Advances in COVID19 Detection, Treatment, and Vaccine Development Useful Conducting the Games in 2021. Advances in Infectious Diseases, 10(03), 56.

Stuart, A. D., Eustace, H. E., McKee, T. A., y Brown, T. D. (2002). A novel cell entry pathway for a DAF-using human enterovirus is dependent on lipid rafts. J.Virol. 76, 9307-9322.

Tian, X., Li, C., Huang, A., Xia, S., Lu, S., Shi, Z., Lu, L. , Jiang , S., Yang, Z., Wu, Y. y Ying, T. (2020). Potent binding of 2019 novel coronavirus spike protein by a SARS coronavirus-specific human monoclonal antibody. Emerging microbes \& infections, 9(1), 382-385.

IPSA SCIENTIA - Vol. 5 No 1 - 2020 
Nistatina como inhibidor de caveolas: tratamiento preventivo para el contagio por COVID-19

Torres, J. R. A., Cutiño, M. P., Prieto, M. R., y González, A. M. (2020). COVID-19 en pediatría: aspectos clínicos, epidemiológicos, inmunopatogenia, diágnóstico y tratamiento. Revista Cubana de Pediatría, 92.

Villafranca, R. C., Albelo, A. N., Moreno, V. F., Pérez, K. B., y Herrera, A. L. G. (2020). SARS-Cov-2, el virus emergente que casusa la pandemia de COVID-19. Revista Médica Electrónica, 42(3).

Weiss, S. R., y Navas-Martin, S. (2005). Coronavirus pathogenesis and the emerging pathogen severe acute respiratory syndrome coronavirus. Microbiol. Mol. Biol. Rev., 69(4), 635-664.

IPSA SCIENTIA - Vol. 5 No 1 - 2020 